\title{
Universal Scaling of Ballistic Magnetoresistance in Magnetic Nanocontacts
}

\author{
S. H. Chung, ${ }^{1}$ M. Muñoz, ${ }^{2}$ N. García, ${ }^{2}$ W. F. Egelhoff, ${ }^{3}$ and R. D. Gomez ${ }^{1, *}$ \\ ${ }^{1}$ Electrical and Computer Engineering Department and Physics Department, University of Maryland, \\ College Park, Maryland 20742 \\ and Laboratory for Physical Sciences, College Park, Maryland 20740 \\ ${ }^{2}$ Laboratorio de Física de Sistemas Pequeños y Nanotecnología, Consejo Superior de Investigaciones Científicas, \\ Serrano 144, E-28006 Madrid, Spain \\ ${ }^{3}$ National Institute of Science and Technology, Gaithersburg, Maryland 20899-8552
}

(Received 14 May 2002; published 27 December 2002)

\begin{abstract}
We show that ballistic magnetoresistance exhibits universal scaling in atomic or nanometer scale contacts. Plotting the data as conductance, we find that, if the maximum magnetoconductance is normalized to unity and the conductance is scaled with the conductivity of the bulk material, the data fall in a narrow region, independent of the nanocontact materials, for our four data sets and four from the literature. The results agree with a theory that takes into account spin-scattering within a magneticdomain wall.
\end{abstract}

DOI: $10.1103 /$ PhysRevLett.89.287203

Ballistic magnetoresistance (BMR) is an effect that occurs in the conduction of spin-polarized electrons through highly constricted $(\sim 10 \mathrm{~nm})$ junctions in which the spin-flip mean free path is long compared with the magnetic domain-wall width. When a magnetic-domain wall resides in the constriction, the electrical resistance is much larger than it is after an external magnetic field is applied to sweep out the domain wall. The resulting magnetoresistive effect is much larger than giant magnetoresistance (GMR) or tunneling magnetoresistance (TMR). Consequently, BMR has the potential to replace TMR in nonvolatile memory chips and replace GMR in hard-disk read heads [1].

Theoretically, there is so far no consensus on the transport mechanism to explain very large values of BMR. Experimentally, there is large variation in the magnitude of the BMR, as well as a wide range of conductances where peak BMR occurs. García et al. observed BMR values up to $\sim 300 \%$ in mechanically formed nanocontacts of various ferromagnetic metals and up to $700 \%$ in electrodeposited $\mathrm{Ni}-\mathrm{Ni}$ nanocontacts [2]. These nanocontacts sometimes exhibit quantized conductance, indicating that the smallest of them consists of only a few atoms. Verluijs et al. [3] observed 540\% BMR in mechanically formed nanocontacts of the half-metallic ferromagnet $\mathrm{Fe}_{3} \mathrm{O}_{4}$. Despite the similar values of the peak BMR in these systems, the resistance regimes in which the maximum BMR is achieved are very different.

In this work, we report the first observations of BMR in $\mathrm{CrO}_{2}-\mathrm{CrO}_{2}$ nanocontacts and in $\mathrm{CrO}_{2}-\mathrm{Ni}$ heterojunction nanocontacts. $\mathrm{CrO}_{2}$ is a half-metallic ferromagnet [4,5]. We also report our own data sets for $\mathrm{Ni}$ and $\mathrm{Fe}_{3} \mathrm{O}_{4}$ nanocontacts. Combining our data and all existing data in the literature $[2,3]$, we show for the first time that the observed $\Delta G / G$ versus the $G$ data (where $G$ is the conductance) all collapse into a narrow region, after appropriate scaling of the conductance for the nanocontact resistivity.
PACS numbers: 75.70.-i, 73.23.Ad, 73.50.-h, 75.25.+z

Thus, the same mechanism, i.e., spin-ballistic transport through the nanocontact, appears responsible for BMR in different regimes of conductance from normal metal $(\mathrm{Ni}$, $\mathrm{Co}, \mathrm{Fe})$, to semimetal $\left(\mathrm{CrO}_{2}\right)$, to an insulator with Verwey transition at $118 \mathrm{~K}\left(\mathrm{Fe}_{3} \mathrm{O}_{4}\right)$ [6]. This implies an universality in the BMR mechanism, indicating that the effect should be observable in a very large class of ferromagnetic material systems.

We performed BMR measurements using the technique of Ref. [2]. Nanocontacts were formed mechanically using a micropositioner, and magnetic fields up to 150 Oe were applied separately by electromagnetic coils wrapped around the Ni rods and driven by ac and dc currents. The ac magnetic field and resistance were simultaneously monitored with a digital oscilloscope. All measurements were carried out at room temperature in air. For $\mathrm{CrO}_{2}$ studies, $200 \mathrm{~nm}$ thick polycrystalline $\mathrm{CrO}_{2}$ films were deposited on the Ni rods. The coercivity of these films is about 45 Oe and the resistivity is about $140 \mu \Omega \mathrm{cm}$. The $\mathrm{Fe}_{3} \mathrm{O}_{4}$ samples were prepared by crushing a $\mathrm{Fe}_{3} \mathrm{O}_{4}$ mineral specimen and attaching sharp $\sim 1 \mathrm{~mm}$ long pieces to the ends of the $\mathrm{Ni}$ rods with conducting epoxy.

Break-junction experiments on both magnetic and nonmagnetic samples were performed using the apparatus. For $\mathrm{Au}, \mathrm{Ni}$, and $\mathrm{Fe}$, a set of plateaus was observed in the conductance $(G)$ near the integer multiples of $G_{0}\left(=2 e^{2} / h\right)$. This effect is typical of the quantized conductance [7]. The plateaus existed for hundreds of milliseconds; conductance fluctuations, a common occurrence in normal metal nanocontacts, were also observed. The oxide nanocontacts showed no quantization plateaus.

The magnetoresistance (MR) of the nanocontact between two $\mathrm{CrO}_{2}$-coated Ni rods, as acquired by the oscilloscope, is presented in Fig. 1. A square wave pattern of an amplitude of \pm 90 Oe was applied on one of the $\mathrm{Ni}$ rods, while the other Ni rod was magnetized to saturation 

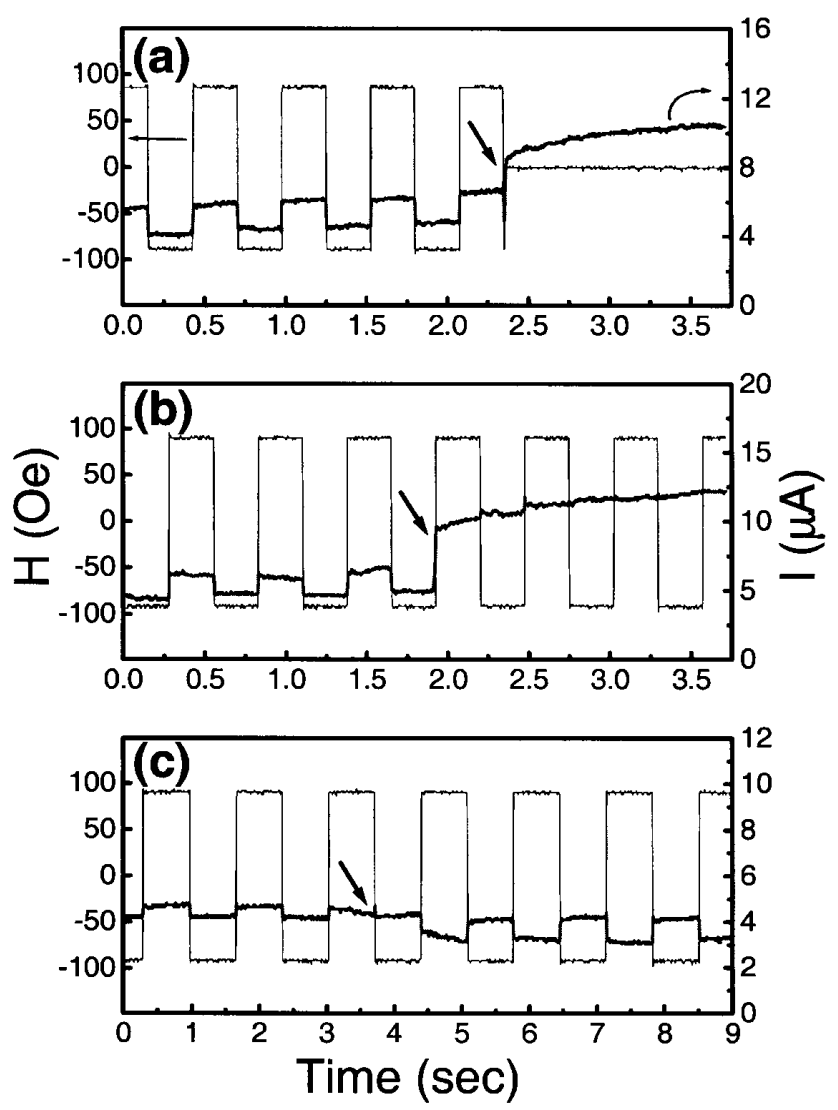

FIG. 1. Typical magnetoconductance data for $\mathrm{CrO}_{2}-\mathrm{CrO}_{2}$ nanocontacts recorded by a digitizing oscilloscope. The thin line shows the ac magnetic field applied to one of the Ni rods. The thick line shows the current through the nanocontact with a bias voltage of $100 \mathrm{mV}$. In (a) the turnoff points of the ac magnetic field are indicated by the arrows. In (b) the turnoff point of the dc magnetic field is indicated by the arrow. In (c) a high external magnetic field is applied opposite to the dc magnetic field at the arrow and a phase inversion of the magnetoconductance data occurs.

by the dc current through the electromagnet. The current across the nanocontact was recorded at a fixed bias of $100 \mathrm{mV}$. The current (and, hence, the resistance of the nanocontact) followed the square wave ac magnetic field with the same phase and frequency. However, when either the ac [Fig. 1(a)] or the dc bias field [Fig. 1(b)] was turned off, no resistance change was observed. Furthermore, upon removal of the dc field [indicated by the arrows in Figs. 1(a) and 1(b)], the current exhibited a rapid transient rise followed by a slow relaxation to higher values.

Several conclusions can be drawn from these measurements. First, the loss of the MR upon turning off the dc field while leaving the ac field on is direct evidence that magnetostriction does not significantly contribute to the observed resistance changes. Otherwise, one would see a modulation of the MR corresponding to the mechanical motion of the ac driven Ni rods. The MR was likewise suppressed when a nonmagnetic $50 \mathrm{~nm}$ Au barrier was placed at the nanocontact between the two $\mathrm{Ni}$ rods.
Second, the fact that both dc and ac fields are necessary implies that a magnetic-domain wall or a large magnetization gradient at the nanocontact is necessary to have MR. With both fields present, the $\mathrm{CrO}_{2}$ magnetic domains on one $\mathrm{Ni}$ rod are pinned by the dc field, while the domains of the other oscillate between \pm saturation. The third conclusion concerns the characteristic transient rise in the current. One can discount the origin of the transient signal from mechanical perturbations, or Faraday induction, as these effects would cause both increases and decreases of the current as well as overshoots. The fact that the transient effect is always positive suggests that upon field removal, the domain wall at the nanocontact is swept away, lowering the resistance. The above observations are further complemented by Fig. 1(c), showing the phase inversion [denoted by the arrow in Fig. 1(c)] of the MR response when a high-field magnet is placed near the dc Ni rod. The phase inversion suggests that the external field is large enough to overcome the dc pinning field of the Ni rod and, consequently, reverse its magnetization. Based on these arguments, the MR response is thus primarily due to the spin-polarized transport between two ferromagnetic reservoirs separated by a large magnetization gradient.

A large number of $\Delta G / G$ versus $G$ measurements were conducted by adjusting the spacing of the Ni rods while continuously monitoring the magnetoconductance (MC). Figure 2 presents the experimental values in the form of plots of MC versus nanocontact conductance. Note that $M C$ values are, by definition, identical in magnitude to $M R$ values. The data on Ni in Fig. 2(a) agree quite well with the results of Ref. [2]. The largest $\mathrm{MC}$ that we observed was $400 \%$ in $\mathrm{CrO}_{2}-\mathrm{CrO}_{2}$ junction at a conductance of $0.05 G_{0}$ [Fig. 2(b)]. By comparison, the maximum $\mathrm{MC}$ of $\mathrm{Ni}-\mathrm{Ni}$ is $210 \%$, which was obtained at $G_{0}$. The behavior of $\mathrm{CrO}_{2}-\mathrm{Ni}$ is similar to the $\mathrm{CrO}_{2}-\mathrm{CrO}_{2}$ in that the peak $\mathrm{MC}$ occurs at very low conductance although the maximum $\mathrm{MC}$ is lower.

A summary of the three cases of Fig. 2 as well as all results from the literature $[2,3]$ are plotted in Fig. 3, which presents the normalized MC as a function of the scaled nanocontact conductance. The normalization was performed for each data set by setting the MC to unity at its peak value. The conductance for each data set was scaled to match that of $\mathrm{Ni}$ by multiplying the actual conductance of each nanocontact by the ratio of the conductivity of $\mathrm{Ni}$ to the conductivity of the other conductor. In the case of the $\mathrm{CrO}_{2}$-Ni heterojunction, we used the conductance of the $\mathrm{CrO}_{2}$.

Figure 3 demonstrates that widely diverse data collapse into a well-behaved functional relationship when scaling is applied. This fact suggests the intriguing possibility that the MC mechanism is the same in all three different systems (metal, semimetal, and Verwey insulator). If so, the physics of the effect is explained by spin conservation in the transport process due to domain-wall scattering at the nanocontact $[8,9]$. 

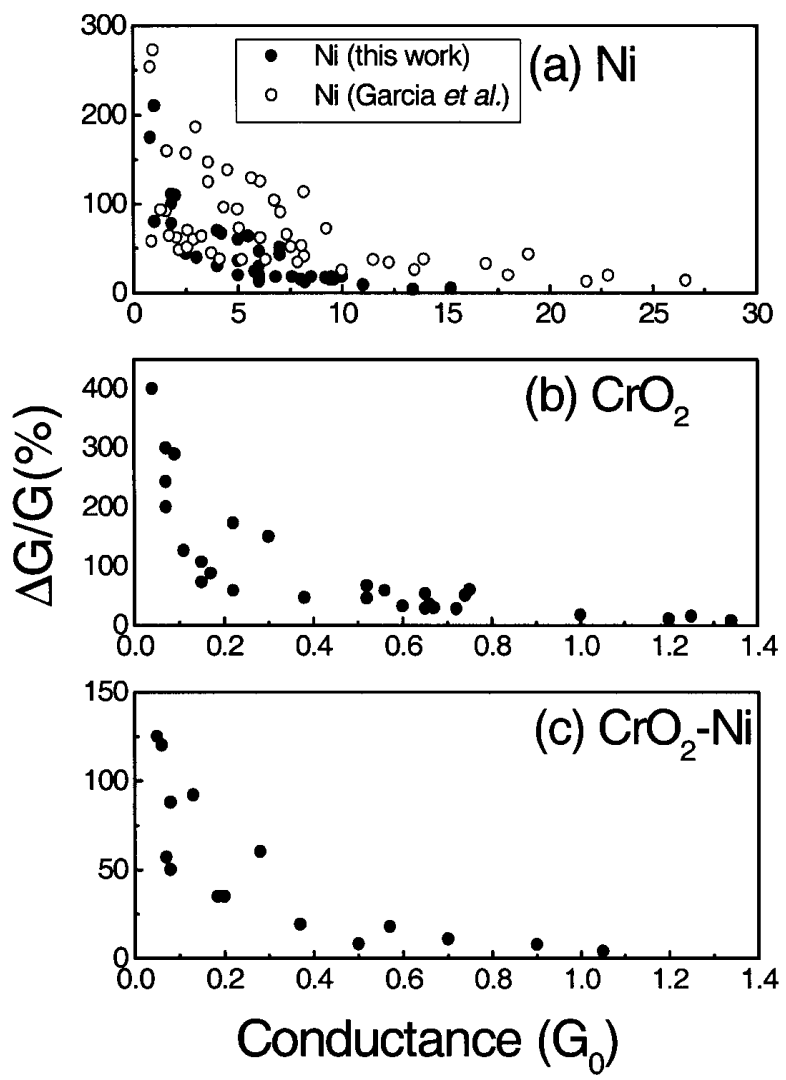

FIG. 2. Experimental data of the magnetoconductance as a function of the nanocontact conductance for (a) $\mathrm{Ni}-\mathrm{Ni}$, (b) $\mathrm{CrO}_{2}-\mathrm{CrO}_{2}$, and (c) $\mathrm{CrO}_{2}-\mathrm{Ni}$ nanocontacts.

The three transition metals $\mathrm{Ni}, \mathrm{Co}$, and Fe have similar resistivities of 6 to $8 \mu \Omega \mathrm{cm}$ and scaling has little effect on the data. The semimetal $\mathrm{CrO}_{2}$ has a resistivity of $140 \mu \Omega \mathrm{cm}$, which is a factor of 23 larger than $\mathrm{Ni}$. We found the best agreement in scaling the $\mathrm{CrO}_{2}$ data to the Ni data occurred using a scaling factor of 19. This small discrepancy is very reasonable, considering that lattice disorder at a mechanically formed nanocontact could easily change the resistivity by a factor of 2 from its bulk single crystal value. However, for the case of the $\mathrm{Fe}_{3} \mathrm{O}_{4}$, the agreement is not as good. $\mathrm{Fe}_{3} \mathrm{O}_{4}$ is an insulator below the $118 \mathrm{~K}$ Verwey transition and at higher temperatures exhibits thermally excited hoping conductivity between the coexisting $\mathrm{Fe}^{3+}$ and the $\mathrm{Fe}^{2+}$. For the data of Verluijs et al. on $\mathrm{Fe}_{3} \mathrm{O}_{4}$, the best agreement is found for a scaling factor of 30 (corresponding to a bulk resistivity of $180 \mu \Omega \mathrm{cm}$ ), and that value was used to plot our $\mathrm{Fe}_{3} \mathrm{O}_{4}$ data in Fig. 3. At room temperature, resistivities from 4000 to $10^{5} \mu \Omega \mathrm{cm}$ have been reported for bulk single crystals and thin films fabricated by a variety of techniques. The reason for the apparent discovery is that $\mathrm{Fe}_{3} \mathrm{O}_{4}$ is complicated and the resistivity values are strongly dependent upon the crystallinity, film thickness, and fabrication conditions. Furthermore, the thermally activated character of the conductance at the nanocontact

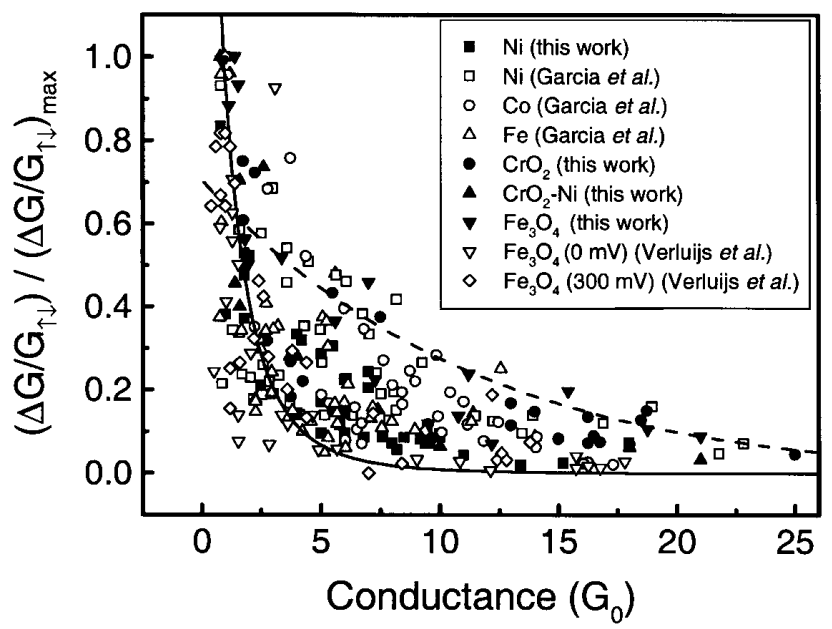

FIG. 3. Normalized magnetoconductance as a function of the nanocontact conductance scaled by the ratio of the material resistivity to the resistivity of $\mathrm{Ni}$. For both our data and that in the literature, the conductances are scaled to $G_{0}$ at the peak magnetoconductance. The solid and dashed lines are from Ref. [8] in the limits of small and large number of conducting channels, respectively.

means that the conductance could easily change by orders of magnitude, unlike in metals.

In the experiments we conducted on $\mathrm{Fe}_{3} \mathrm{O}_{4}$ using the same procedures as in Ref. [3], we obtain maximum MC of $350 \%$, consistent with that of Ref. [3]. However, in our case, the MC peaked at much lower conductance and the scaling factor of 200 gives the best agreement. This scaling factor was used to plot their data in Fig. 3. This result would imply that in our case, the resistivity at the nanocontact is about $1400 \mu \Omega \mathrm{cm}$, which is closer to values reported for bulk $\mathrm{Fe}_{3} \mathrm{O}_{4}$.

The scaling with the resistivities is the key point in explaining the universality of BMR. According to the Cabrera and Falicov theory [9] of the scattering of electron spins by a domain wall (DW) as discussed by Tatara et al. [8] for nanocontacts, the spin transport adiabaticity increases as the DW width increases. In thick DW's the MR is reduced, as the spin can rotate to align with the local magnetization as the electron travels through the wall. Conversely, for very thin domain walls $(\approx 1 \mathrm{~nm})$ the spin is almost completely conserved as the electron crosses the wall. From Refs. [7-9], the conductance for the parallel magnetization configuration of the Ni rods, with no DW at the nanocontact, is given by the Landauer formula [10]:

$$
G_{\Uparrow}=\frac{2 e^{2}}{h} \sum_{i} T_{i},
$$

where $e$ and $h$ are the electron charge and Planck's constant, respectively. $T_{i}$ is the transmissivity of the channel $i$ at the nanocontact for spin-up electrons. The conductance for the antiparallel magnetic configuration is $G_{\uparrow \downarrow}=R^{-1}$, where the resistance $R$ is 


$$
R=G_{\Uparrow}^{-1}+R_{w},
$$

and the DW resistance $R_{w}$ for nanocontact of the same metal is

$$
R_{w} \approx \frac{1}{G_{\uparrow}} \frac{2 P^{2}}{1-P^{2}} F(P, \lambda)
$$

where $P=\frac{D_{\uparrow}-D_{\downarrow}}{D_{\uparrow}+D_{\downarrow}}$ is the polarization at the Fermi level given by the density of states ratio of $D_{\uparrow}$ and $D_{\downarrow} ; \lambda$ is the DW width.

The relative conductance is

$$
\frac{\Delta G}{G_{\uparrow \downarrow}}=\frac{G_{\Uparrow}-G_{\uparrow \downarrow}}{G_{\uparrow \downarrow}}=\frac{2 P^{2}}{1-P^{2}} F(P, \lambda),
$$

which is independent of the conductance. $F(P, \lambda)$ is the function that gives the accommodation of the spin in the DW [8,9]. Note that this is unity for $\lambda \rightarrow 0$, and Eq. (4) reduces to Julliere's [11] result when the spin transport is completely nonadiabatic, i.e., the spin is conserved. On the other hand, $F(P, \lambda) \rightarrow 0$ as $\lambda$ grows. This function behaves as $\exp (-\beta \lambda)$ for $\lambda \approx \lambda_{F}$ and as $\frac{1}{\alpha \lambda^{2}}$ for $\lambda \gg \lambda_{F}$, where $\lambda_{F}$ is the Fermi wavelength and $\beta, \alpha$ are constants $[8,9]$. Making the reasonable assumption that $\lambda \sim d(d$ is the nanocontact cross-section diameter), then $\lambda \sim N^{1 / 2}$ where $N$ is the number of conducting channels allowed in the nanocontact. The normalized result for the $\mathrm{Ni}-\mathrm{Ni}$ nanocontacts is presented in Fig. 3 by the continuous line for small $N$ and by the dashed line for the asymptotic value for $N$ large.

It is thus clear that the behavior of the normalized MC is defined by the spin-scattering function $F(P, \lambda)$ $[8,9,12,13]$. The value of the conductance can be obtained by using Sharvin's formula [14]:

$$
G=G_{0} \frac{d^{2}}{\lambda_{F}^{2}} \gamma
$$

where $\frac{d^{2}}{\lambda_{F}^{2}}$ is the number of channels and $\gamma$ the transmissivity per channel. The interpretation of the data using Eq. (5) takes into account the different material resistivities, and this is our approach to establishing the general behavior of the data. The point is that the MR does not need only ballisticity to have the universal behavior. Diffusive, or activated, etc., transport can also exhibit the same universal behavior, because at the end of the nanocontact at which the DW scattering occurs, the channel transmissivity depends on the transport process and thus on the resistivity. However, the ratio of the currents in the parallel and antiparallel configurations does not depend on how large the transmissivities are but is instead defined by the DW scattering. Therefore, if $\gamma \ll 1$, then the conductance can be much smaller than $G_{0}$ even if the number of channels is larger than 1 . As the $F(P, \lambda)$ is scaled by $\lambda \sim d$, then from Eq. (5) it is clear that $\lambda$ can be large while $G$ is small and that $\gamma$ is the scaling factor that is approximately inversely proportional to the resistivity of the material.
We note that the BMR results of Ref. [2] for electrodeposited nanocontacts belong to a different ensemble than the data described here. In such cases, the nanocontact contains a very thin $(<1 \mathrm{~nm})$ metallic dead magnetic layer that electrons can transit with spin conservation $(F=1)$. In such a case, there is no domain wall, but an abrupt change in the magnetization on opposite sides of the dead layer occurs.

In conclusion, we have shown that all the existing MR data on mechanically formed nanocontacts behave universally for the normalized MR versus conductance, when scaled according to the resistivity of the material. The behavior is completely determined by the spinscattering at the domain wall and controlled by the domain-wall thickness only. The conductance is proportional to the area of the nanocontact times the transmittance of the nanocontact. The transmittance of the nanocontact is approximately inversely proportional to the bulk resistivity of the material.

This work is supported under NSF Grants ECS 0115327, ECS9984797 CAREER Award, and by the Spanish DGICyT. Support from UMCP NSF-MRSEC is also gratefully acknowledged.

*Corresponding author.

[1] G. Prinz, Science 282, 1660-1663 (1998); E. Y. Tsymbal and D. G. Pettifor, Solid State Phys. 56, 113 (2001).

[2] N. García, M. Muñoz, and Y-W. Zhao, Phys. Rev. Lett. 82, 2923 (1999); Appl. Phys. Lett. 76, 2586 (2000); N. García, M. Muñoz, G. G. Qian, H. Rohrer, I. G. Saveliev, and Y.-W. Zhao, Appl. Phys. Lett. 79, 4550 (2001).

[3] J. J. Verluijs, M. A. Bari, and J. M. D. Coey, Phys. Rev. Lett. 87, 026601 (2001).

[4] K. Schwarz, J. Phys. F 16, L211 (1986); R. A. de Groot and F. M. Mueller, Phys. Rev. Lett. 50, 2024 (1983).

[5] R. J. Soulen et al., Science 282, 85 (1998).

[6] S. Chikazumi, Physics of Ferromagnetism (Clarendon, Oxford, 1997), 2nd ed., p. 205.

[7] J. I. Pascual, J. Méndez, J. Gómez-Herrero, A. M. Baró, N. García, and Vu Thien Binh, Phys. Rev. Lett. 71, 1852 (1993).

[8] G. Tatara and H. Fukuyama, Phys. Rev. Lett. 72, 772 (1994); G. Tatara, Y.-W. Zhao, M. Muñoz, and N. García, Phys. Rev. Lett. 83, 2030 (1999).

[9] G. G. Cabrera and L. M. Falicov, Phys. Status Solidi B 61, 539 (1974).

[10] R. Landauer, Philos. Mag. 21, 863 (1970).

[11] M. Julliere, Phys. Lett. 54A, 225 (1975).

[12] N. García, Appl. Phys. Lett. 77, 1351 (2000); G. G. Cabrera and N. García, Appl. Phys. Lett. 80, 1782 (2002).

[13] J. B. A. N. H. van Hoof, K. M. Schep, A. Brataas, G. E.W. Bauer, and P. J. Kelly, Phys. Rev. B 59, 138 (1999).

[14] Yu.V. Sharvin, Zh. Eksp. Teor. Fiz. 48, 984 (1965) [Sov. Phys. JETP 21, 655 (1965)]. 